\title{
Solving open travelling salesman subset-tour problem through a hybrid genetic algorithm
}

\author{
Purusotham Singamsetty $^{\mathrm{a}}$, Jayanth Kumar Thenepalle ${ }^{\mathrm{b}^{*}}$ and Balakrishna Uruturu ${ }^{\mathrm{b}}$
}

${ }^{a}$ Department of Mathematics, School of Advanced Sciences, VIT, Vellore-632014, Tamil Nadu, India

${ }^{b}$ Department of Science and Humanities, Sreenivasa Institute of Technology and Management Studies, Chittoor-517127, Andhra Pradesh, India

\section{H R O N I C L E}

Article history:

Received: November 30, 2020

Received in revised format:

April 2, 2021

Accepted: May 2, 2021

Available online:

May 5, 2021

Keywords:

Travelling salesman problem

Open travelling salesman subset-

tour problem

Genetic algorithm

Complex mutation

\section{A B S T R A C T}

In open travelling salesman subset-tour problem (OTSSP), the salesman needs to traverse a set of $k(\leq n)$ out of $n$ cities and after visiting the last city, the salesman does not necessarily return to the central depot. The goal is to minimize the overall traversal distance of covering $k$ cities. The OTSSP model comprises two types of problems such as subset selection and permutation of the cities. Firstly, the problem of selection takes place as the salesman's tours do not contain all the cities. On the other hand, the next problem is about to determine the optimal sequence of the cities from the selected subset of cities. To deal with this problem efficiently, a hybrid nearest neighbor technique based crossover-free Genetic algorithm (GA) with complex mutation strategies is proposed. To the best of the author's knowledge, this is the first hybrid GA for the OTSSP. As there are no existing studies on OTSSP yet, benchmark instances are not available for OTSSP. For computational experiments, a set of test instances is created by using TSPLIB. The extensive computational results show that the proposed algorithm is having great potential in achieving better results for the OTSSP. Our proposed GA being the first evolutionary-based algorithm that will help as the baseline for future research on OTSSP.

\section{Introduction}

The travelling salesman problem (TSP) is one of the broadly considered combinatorial optimization routing problems that was first coined by two mathematicians in the 1800s (Matai et al., 2010), and then formulated by Karl Menger (Maredia, 2010). In TSP, a set of cities and a salesman will be given. The salesman is intended to cover all the given cities and come back to the city where he started. The objective is to cover all the given cities and come back to the home city with overall least traversal distance. However, the basis of the TSP is aimed to determine a closed Hamilton path that means a path that covers every node/city in the graph exactly once and comes back to the starting point, the works on Open Travelling SalesmanSubset Tour Problem (OTSSP) is limited. Yet, in the practical transportation scenarios, the salesman need not necessarily cover all the given $n$ cities, but only enough to cover $k$ cities from the given $n$ cities. The OTSSP can be described as follows: Given a set of $n$ cities including a starting/home/depot city and a predetermined value $k(\leq n)$, the OTSSP aims to find a subset of $k$ cities having the depot city, where the salesman starts the tour, cover each city from this subset exactly once and not necessarily to come back to the depot city such that the overall traversal distance. In other words, the OTSSP consists of selecting a subset with exactly $k$ out of $n$ cities and sequencing them to minimize the overall traversal distance by the salesman. This model has wide practical utility in those situations like when there are inadequate resources to cover all the given cities and the organizations working together with outsourcing agencies. More often, this scenario can be seen in rural healthcare servicing and design of distribution networks. For instance, if the logistic distribution agency taking services from an outsourcing agency is not having sufficient resources to cover all the cities, it is significant to serve partial cities. Thus,

* Corresponding author.

E-mail address: jayanth.maths@gmail.com (J. Thenepalle) 
the salesman starts from the depot city, covers only a limited number of cities instead of all the cities and need not return to the home city at the end with the least possible distance. Figures 1,2 and 3 demonstrate the difference between classical travelling salesman problem (TSP), travelling salesman subset tour problem (TSSP) and open travelling salesman subset tour problem (OTSSP), respectively. Fig. 1 represents the classical TSP in which the salesman starts from a depot city, covers the rest of the given 7 cities exactly once and comes back to the home city with minimum overall traversal distance. Fig. 2 depicts an arbitrary solution of TSSP in which the salesman starts from the depot city, needs to cover only $k=6$ out of 8 cities and returns to the depot city. Finally, Fig. 3 indicates an arbitrary solution of OTSSP, where the salesman starts from the depot city, needs to cover only $k=6$ out of 8 cities and not necessarily to come back to the depot city.

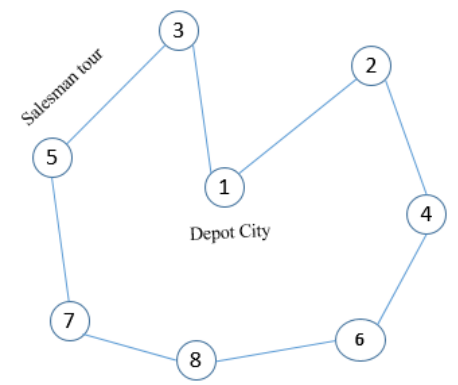

Fig. 1. An example solution of TSP with $n=8$ cities

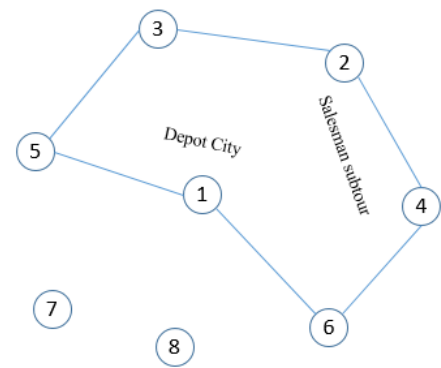

Fig. 2. An example solution of TSSP with $k=6$ cities

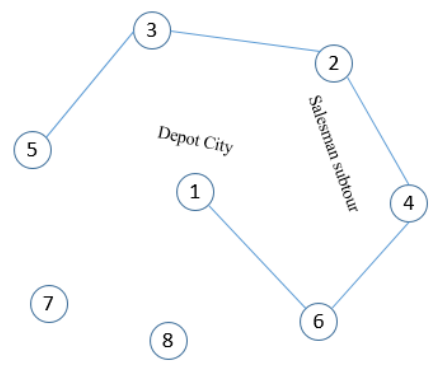

Fig. 3. An example solution of OTSSP with $k=6$ cities

The classical TSP can be seen as a special case of TSSP with $k=n$ and can be seen as OTSSP in the presence of closed tour and $k=n$. On the other hand, TSSP in itself can be seen as an exceptional case of prize collecting travelling salesman problem (PCTSP) (Balas, 1989) in which each city is specified with a prize as well as a penalty. When the salesman visits any city, he will accumulate the prize corresponding to the visited city and if he does not visit any city, then a penalty will be incurred related to the unvisited city. The objective of the PCTSP is to minimize the overall traversal distance covered by the salesman and the net penalties acquired while accumulating a specified minimum total of the prize. When penalties for every city is assumed to be zero, the PCTSP becomes the quota travelling salesman problem (QTSP). When we assume the prize assigned to each city is 1 and the specified minimum total of the prize is $k$, then the model QTSP can turn into TSSP (Ausiello et al., 2018). Since the classical TSP is NP-hard, it is obvious that the variants namely TSSP and OTSSP are also NP-hard. To best of author's knowledge, Chieng \& Wahid, (2014) first addressed and developed a genetic algorithm to solve OTSSP model. We address the same problem OTSSP and develop a hybrid nearest neighbour based genetic algorithm. Reviewing the literature, the work on OTSSP is still very limited. The TSSP is a special case of OTSSP, where the salesman need not necessarily return to the starting city. Concerning the literature, the TSSP is also referred to as $k$-TSP (Venkatesh et al., 2018). To review the earlier works, Saksena and Kumar (1966), Ibaraki (1973) and Laporte et al. (1984) studied TSSP with an additional constraint that the salesman has to visit a subset of $k(\leq n)$ specified nodes. A variety of solution techniques including heuristics and exact algorithms for TSSP and its variants can be found in the literature. Gensch (1978) modelled TSSP as an industrial scheduling problem with an additional time constraint. In this variant, the salesman needs to cover only a subset of the cities from a given set of cities with minimum distance without violating the time limit. To solve this problem, the Lagrangian relaxation-based branch and bound algorithm have been developed that deals problems of a practical size. Mittenthal and Noon (1992) proposed an insertion/ deletion based heuristic algorithm to solve TSSP with an additional constraint efficiently and its effectiveness is shown through experimental results. Verweij and Aardal (2003) discussed the merchant sub tour problem whose objective is to find the closed tour that maximizes the profit over a vertex and edge-weighted complete graph and developed a linear programming approach for its solution. Westerlund et al. (2006) addressed TSSP that finds a path from a depot on the node and edge-weighted undirected graph with prizes and Knapsack limitations on the node weights. A heuristic decomposition scheme-based column generation algorithm was developed to solve this problem.

Giardini and Kalmár-Nagy (2011) studied sub tour problem and it has been applied to multiagent planning scenarios. To tackle this model, a hybrid Genetic algorithm (GA) combined with a local search algorithm has been developed and has demonstrated the algorithm's efficiency through experimental results. Stetsyuk (2016) has given the statement of $k$-node shortest cycle in a complete weighted graph and formulated as a Boolean linear programming problem. This study has experimentally shown that the problem of determining the $k$-node shortest cycle is more challenging than the problem of identifying the shortest Hamiltonian cycle. This is due to the solution consisting in finding the optimal subset of nodes corresponding to the shortest Hamiltonian cycle. Venkatesh et al. (2018) considered $k$-TSP and proposed a first simple and efficient metaheuristic algorithm called general variable neighborhood search algorithm (GVNS) to solve TSSP/ $k$-TSP. This technique combines two neighborhood strategies such as exchange and swap processes, which effectively accomplish both the features such as subset selection and permutation of the cities of the $k$-TSP. More recently, Venkatesh et al. (2020) has developed two multi-start heuristic algorithms, which combines a GVNS approach and hyper-heuristic for solving $k$-TSP. From the literature, of all the metaheuristic approaches, Genetic algorithm (GA) has proven to have good capability in dealing combinatorial optimization problems (Bahaabadi et al., 2012). The former-cited works motivate to address the practical variant of TSP called 
OTSSP. Solving the OTSSP includes two features, namely subset selection and permutation. Here, subset selection is itself a problem of picking a subset that contains $k$ out of $n$ cities with depot city as well and permutation means the problem of determining the best permutation of the $k$ cities from the selected subset. Solution methods for OTSSP have to address both of these features in a suitable way to tackle a wide range of test instances effectively. If the approach to deal with the subset selection feature is not project appropriately, it will not produce the best solutions for OTSSP and it does not matter how best the approach is designed for permutation feature. Similarly, an approach with a good scheme to tackle permutation features but a weak scheme for subset selection feature, will not yield the best solutions either. Hence, for any solution approach to solve OTSSP, relative importance should be given to these two aspects to getting the best solutions. By understanding its importance, we have developed a hybrid nearest neighbor technique based Genetic algorithm with complex mutation operators for OTSSP. The main contribution of this study is addressing a practical variant namely OTSSP and development of a hybrid Genetic algorithm (GA) that effectively solves OTSSP. Generally, the initial population is produced randomly. Since the OTSSP solutions do not include all the cities, GA through randomly generated population may result in solutions far away from the optimal or near-optimal solutions. Hence, an efficient systematic procedure is essentially required to generate the initial population. However, in this study, we propose an efficient nearest neighborhood algorithm that effectively generates relatively good initial population. Further, to make the GA converge toward optimal or near-optimal solutions, complex mutation operators that include a slide, swap, reverse swap, and other combinations being considered. To the best of author's knowledge, the proposed GA is the first evolutionary hybrid algorithm for OTSSP.

The rest of the paper is organized as follows: The following section will provide the definition and formulation of the OTSSP. Section 3 will give an outline of the GA and its operators. Section 4 demonstrates the computational results. Finally, the conclusion and scope of future work are described in Section 5.

\section{Problem statement and mathematical formulation}

Let $G=G(V, E)$ be the complete, undirected, edge-weighted graph, where the node-set $V=\{1,2, \ldots, n\}$ denotes a set of $n$ cities including one central depot /home city/starting city and the edge set $E=\{(i, j) / i, j \in V ; i \neq j\}$ be the set of $n^{2}-n$ edges. Note that the terms node and city are synonymously used hereafter. Each node is specified with a position $\left(x_{i}, y_{i}\right)$ in the Cartesian coordinate system. Each edge $(i, j)$ is associated with a distance/cost $d_{i j}=\sqrt{\left(x_{i}-x_{j}\right)^{2}+\left(y_{i}-y_{j}\right)^{2}} ; \quad\left(d_{i j}=d_{j i} ; d_{i i}=\infty, d_{i j}>0\right)$, which is the Euclidean distance between the cities $i$ and $j$. Let the salesman be positioned at the starting city/ central depot. The salesman need to cover a subset $S(|S|=k$, where $S \subseteq V)$ of $k(\leq n)$ cities starts from the home city, takes a route by covering the rest of the $k-1$ cities of $S$ exactly once and do not necessarily return to the home city. The possible sub tours induced on covering these $k$ cities will be $\left(\begin{array}{l}n \\ k\end{array}\right) \times(k-1) !$. The OTSSP aims to determine an optimal open path that covers $k$ out of $n$ cities, such that the overall traversal distance is minimized. Note that, a path of length $k$ is said to be a feasible solution and an infeasible solution, otherwise. Here, the binary variable $x_{i j} \in\{0,1\}$, such that $x_{i j}=1$ if the salesman visits $j^{\text {th }}$ city from $i^{\text {th }}$ city, and $x_{i j}=0$, otherwise. Here, another binary variable $y_{j} \in\{0,1\}$ is introduced, such that $y_{j}=1$, if the $j^{\text {th }}$ city is included in the subset and $y_{j}=0$, otherwise. Note that the starting city is assumed as city 1 in this study. The mathematical model for OTSSP is as follows:

$$
\min Z=\sum_{i=1}^{n} \sum_{j=1}^{n} d_{i j} x_{i j}
$$

subject to:

$$
\begin{aligned}
& \sum_{j=2}^{n} x_{1 j}=1 \\
& \sum_{i=2}^{n} x_{i 1}=0 \\
& \sum_{i=1}^{n} x_{i j} \leq 1 ; \forall j \in V \& i \neq j
\end{aligned}
$$




$$
\begin{aligned}
& \sum_{j=1}^{n} x_{i j} \leq 1 ; \forall i \in V \& i \neq j \\
& x_{1 p_{1}}+x_{p_{1} p_{2}}+x_{p_{2} p_{3}}+\ldots+x_{p_{i-1} p_{i}}=k-1 ; p_{1}, p_{2} \ldots p_{i} \in V /\{1\} \\
& \sum_{i=1}^{n} x_{i j}-\sum_{p=2}^{n} x_{j p} \leq 1 ; \forall j \in V \\
& \sum_{i=1}^{n} y_{i}=k \\
& x_{i j}, y_{i} \in\{0,1\} ; \forall(i, j) \in E ; i \in V
\end{aligned}
$$

The objective function (1) represents the minimization of the overall traversal distance on touring $k$ cities by the salesman. Constraint set (2-3) indicates that the salesman starts from the home city and not necessarily returns to the home city. Constraint set (4-5) ensures that the salesman can visit a city and departs from that city at most once. A Hamiltonian path with length $k-1$ involves precisely $k-1$ edges and $k$ cities, thus the Constraint (6) is enforced to guarantee that there are $k-1$ edges. However, this constraint does not guarantee the construction of the feasible path with those $k-1$ edges (For instance, if $n=9$ and $k=4$, one can select the 3 -edges as $(1,3),(3,2),(6,5)$, which cannot build a feasible path of length 3 . Furthermore, the cities involved in the subset $S=\{1,3,2,5,6\}$ violate the desired cardinality i.e. $|S|=k \neq 4$. The trip must be a continuous path that starts at the home city and should cover exactly 4 cities including the home city. The degree of each city must be two (except the last city) in the path (one in degree and one out-degree). To maintain this, Constraint (7) has been introduced. Hence, it preserves the degree constraint for each city, except the last city in the path. Constraint (8) represents a feasible tour that covers exactly $k$ cities. Finally, in Constraint (9), $x_{i j}$ and $y_{j}$ represents the decision variables that take binary values.

\section{Genetic algorithm}

In this section, first, the basic Genetic algorithm (GA) is described, and then the proposed algorithm is discussed in detail. The GA is one of the extensively used metaheuristic algorithms in evolutionary computation for solving combinatorial optimization problems (Goldenberg, 1989). This algorithm was first coined by Holland in 1975, which is an adaptive searching technique based on the survival of the fittest strategy. In its nature, the GA starts with a set of initial solutions/individuals called the initial population, also referred to as chromosomes, in which all the genetic data is stored. Each number within the chromosome is considered as a gene. Further, a fitness value is determined to evaluate the performance of a chromosome. Each time, two chromosomes, called parent chromosomes are selected from the population randomly, which is proportionate to their fitness value. Then, the two chromosomes perform crossover to produce two new chromosomes for the subsequent generation. These new chromosomes will swap old ones if they have superior fitness values. Then, a mutation operation is applied to the newly generated chromosomes to preserve the diversity of the population. Repeat selection, crossover, and mutation processes to generate more chromosomes that are new until the newly generated population size equals the old one. The iteration then starts with the new population. Since better chromosomes will always have a higher chance of being selected for crossover and the new chromosomes generated to transmit the characteristics of their parent chromosomes. The search process continues for many generations until stopping criteria are met. Thus, the entire process is called classical GA. However, there are certain studies in which GA without crossover has been developed. For instance, Liu \& Kroll, (2016) studied multi-robot task allocation problem and a crossover-free GA with complex mutation operators (slide, inversion, swap, insertion, and other combinations) has been presented and showed that the crossover-free GA finds better results than that of the classical GA. To solve OTSSP via GA effectively, the key elements such as chromosome representation, population initialization, fitness evaluation, selection, crossover, mutation operators and GA parameters are required. Different GA strategies have distinct encoding, crossover and mutation operators, which results in divergence of the search process. Thus, it is inevitable to redesign the above operations to confirm that the optimal/suboptimal solution is indeed achieved. To solve OTSSP effectively, a crossover-free GA with complex mutation operators (swap, slide, reverse swap) is developed. The key elements involved in the proposed GA for solving OTSSP are described in the following subsections.

\subsection{Encoding}

The practice of genetic encoding is significant for producing feasible chromosomes. The strategies for encoding chromosomes vary from problem to problem and consists of a certain extent of art. For the travelling salesman problem (TSP), the solution is often indicated as a chromosome of length with the cities involved in the problem. Reviewing the literature, path representation is widely used in solving TSP and its variants (Hussain et al., 2017). In path representation, each chromosome is expressed by an arrangement of $n$ distinct integers. To represent OTSSP solution, the present study utilizes a modified path representation in which a chromosome consists of $k-1$ genes alone (since OTSSP solution involves only $k$ cities). A chromosome can often be represented as $\left(g_{1}, g_{2}, \mathrm{~g}_{3}, \ldots g_{k-1}\right)$, where $g_{j} \in V /\{$ home city $\}, 1 \leq j \leq k-1$ and each $g_{i}$ indicates a gene (city) in the chromosome. This kind of chromosome representation with length $k$ is easy to implement and interpret as 
OTSSP solution. For instance, if $n=8, k=6$, then an arbitrary OTSSP solution corresponding chromosome is $(7,2,4,6,3)$ and same is shown in Fig. 4. The resultant feasible path $1-7-2-4-6-3$ can be returned from the chromosome by affixing the home city at the beginning.

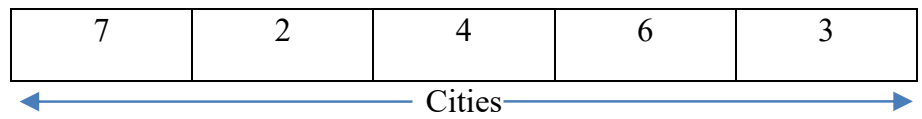

Fig. 4. An arbitrary OTSSP solution with 6 out of 8 cities

\subsection{Initial population}

A finite collection of feasible chromosomes is generally called the initial population and its generation plays a vital role in the GA. This pool consists of valid chromosomes, which are usually generated randomly. Since each of OTSSP solutions involves only $k$ out of $n$ cities, therefore identifying which $k$ cities would result in the optimal or near-optimal solution is still a challenging task. Hence, a well-sophisticated technique is essentially needed to generate the initial population. In this study, the initial population is efficiently generated to assure better and faster convergence in producing the optimal or near-optimal results. The initial population for the proposed GA was created using the nearest neighbor algorithm. It is obvious that the best solution chromosome certainly includes minimum distance edges. With this fact, first, the elements of the distance matrix will be sorted ascending order along with their corresponding indices. By selecting the least distance corresponding first node and using the nearest neighbor heuristic, first, the chromosome is generated. In the same way, select the next least distance corresponding to the first node and apply the nearest neighbor heuristic, the second chromosome is produced. In such a way, for $n \times n$ symmetric matrices, a set of $\left(n^{2}-n\right) / 2$ valid non-redundant chromosomes can be generated. Of which, only the desired number of best chromosomes can be chosen for further process. The pseudo-code of the nearest neighbor heuristic is presented in Algorithm 3.1. The chromosome demonstrated in Fig. 4, represents a single individual of the population called one of the solutions of the problem.

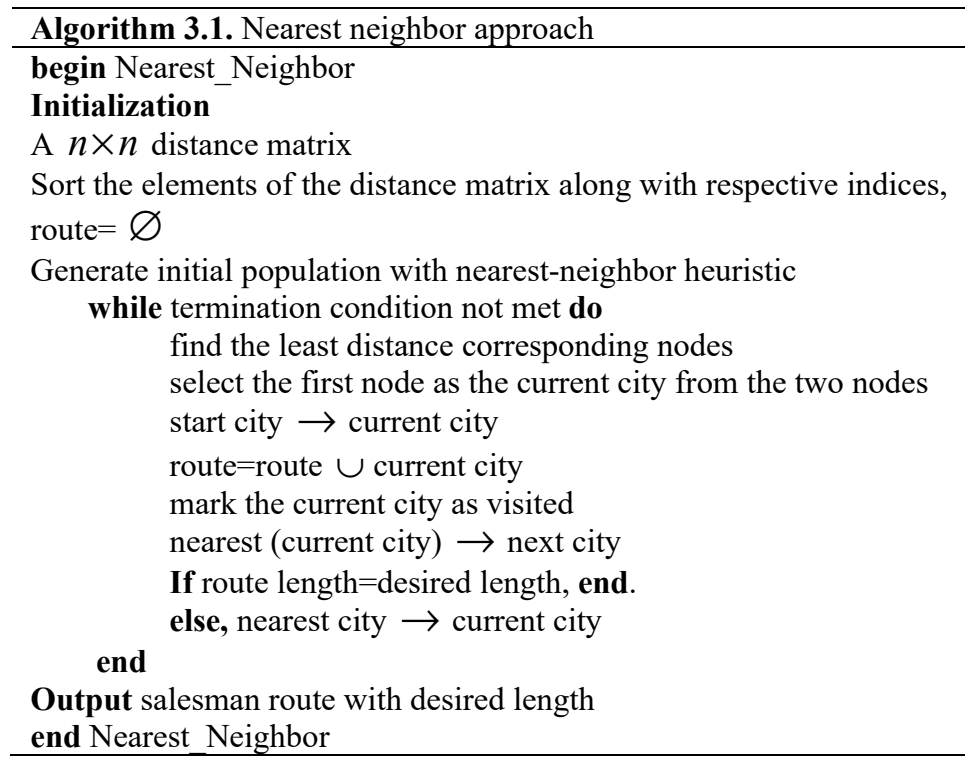

\subsection{Fitness function}

The fitness function helps to evaluate candidate chromosomes in the population. In our study, the fitness function is considered as the objective function given in Eq. (1). Therefore, the chromosome with smaller distance/cost will have a higher fitness value and thus have a greater genetic probability to be selected. For the OTSSP, the fitness value represents the overall distance of the salesman on covering exactly $k$ cities including the home city.

\subsection{Selection}

The selection operator is another significant step in the GA, which helps to create a new population with higher fitness value from the current population. Its main intention is to carry the high-quality genes to the subsequent generation and to improve the efficiency of evaluation and convergence towards the optimal and near-optimal solution. The present study uses the 
classical roulette wheel strategy to the selection operation. This operator selects a chromosome from its population in a statistical fashion depending on its fitness value to enter into a reproducing pool. Those chromosomes closer to the solution have a better chance of being selected.

\subsection{Mutation operator}

Mutation operation is performed next to the selection. It avoids the GA from being trapped in a local optimum and enhances the genetic variability of the population. This work utilizes the complex mutation operator, which comprises Swap, Reverse swap/Flip and Slide mechanisms. All these mutation operators are incorporated to get optimal solutions or near-optimal solutions in a limited time. With a mutation probability $P_{m}$, a parent chromosome is chosen. For a swapping operation, two different positions are chosen randomly from the parent chromosome; the genes of these two positions are interchanged. For a reverse swap operation, two different positions are chosen to describe segments, the genes between these positions are reversed. Similarly, for a slide operation, two distinct positions are selected (say, $i^{\text {th }}$ and $j^{\text {th }}$ positions). The new offspring can be produced by removing the gene in $i^{\text {th }}$ position and copy the same in $j^{\text {th }}$ position of the parent chromosome. Thus, genes between $i^{\text {th }}$ and $j^{\text {th }}$ positions will be decremented by one, i.e. the gene at $(i+1),(i+2)$ positions will be moved to $i^{t h}$ and $(i+1)^{\text {th }}$ positions, respectively and so on. Similarly, the gene at $i^{\text {th }}$ position will be moved to $j^{\text {th }}$ position and the gene associated at $j^{\text {th }}$ position should be moved to $(j-1)^{\text {th }}$ position. Examples for Swap, Reverse swap/Flip and Slide operations are illustrated in Figs. 5-7, respectively.

\subsection{GA parameters}

In addition to the key elements of GA discussed earlier, setting appropriate values to the parameters namely, size of the population, mutation probability rate and termination criteria also plays a vital role in the algorithm's efficiency. These parameters are varied by the problem to be tackled. The population size indicates the number of chromosomes in any one generation and it is considered sufficient as large as 100 in this study. Although crossover operators are not considered in this study, with complex mutation strategies the diversity in the population can be achieved. Mutation probability rate $\left(P_{m}\right)$ indicates how frequently the mutation operation is performed to the parts of the chromosome. It makes changes in the part of the chromosomes and thus maintains the diversity in the population. Generally, $P_{m}$ lies between 0.001 and 0.1 . In our study, it is considered as 0.01 . Finally, the termination criterion of the GA is assumed to be a maximum number of generations. The process of the proposed GA is demonstrated in Fig. 8.

Parent:

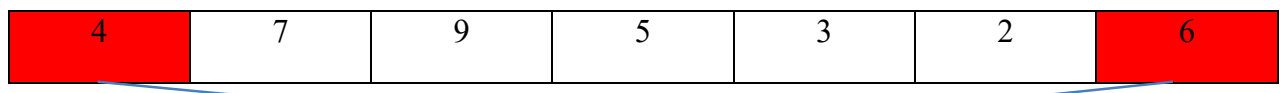

Child

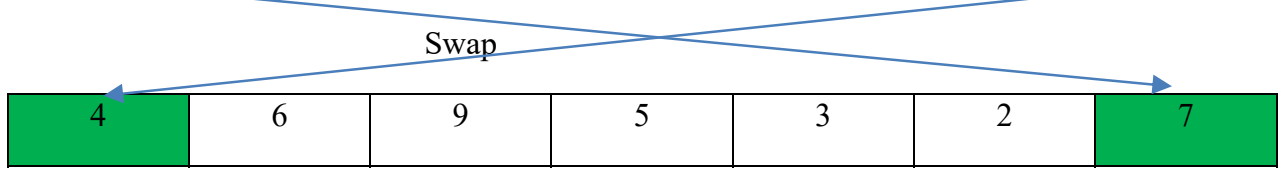

Fig. 5. Swap Operator

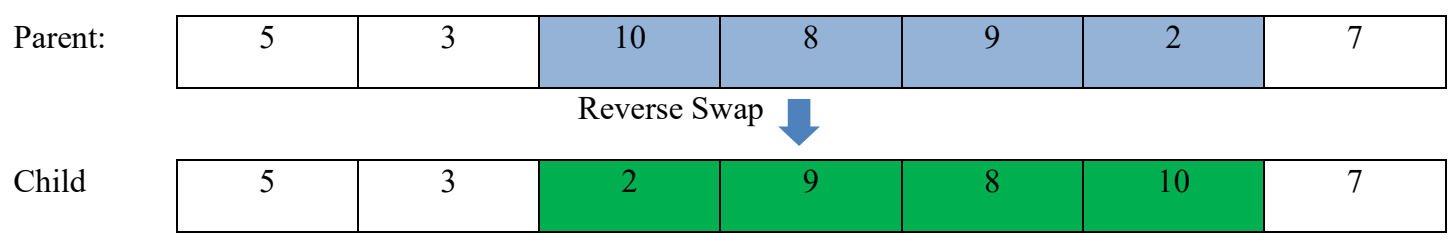

Fig. 6. Reverse Swap Operator

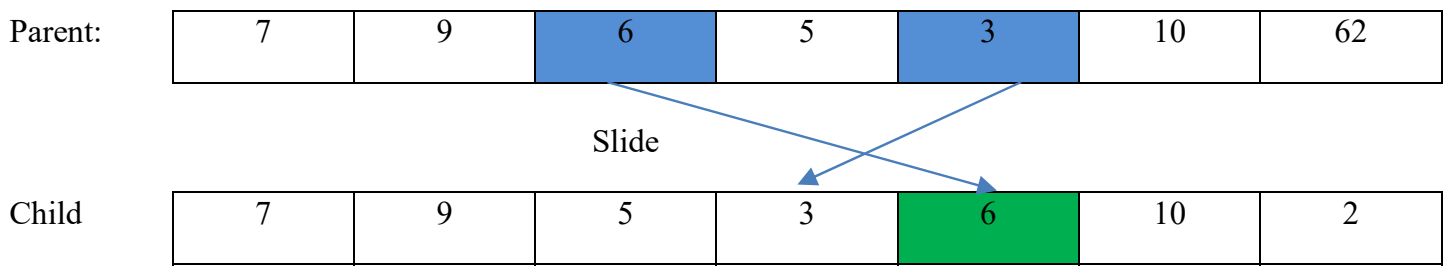

Fig. 7. Slide Operator 


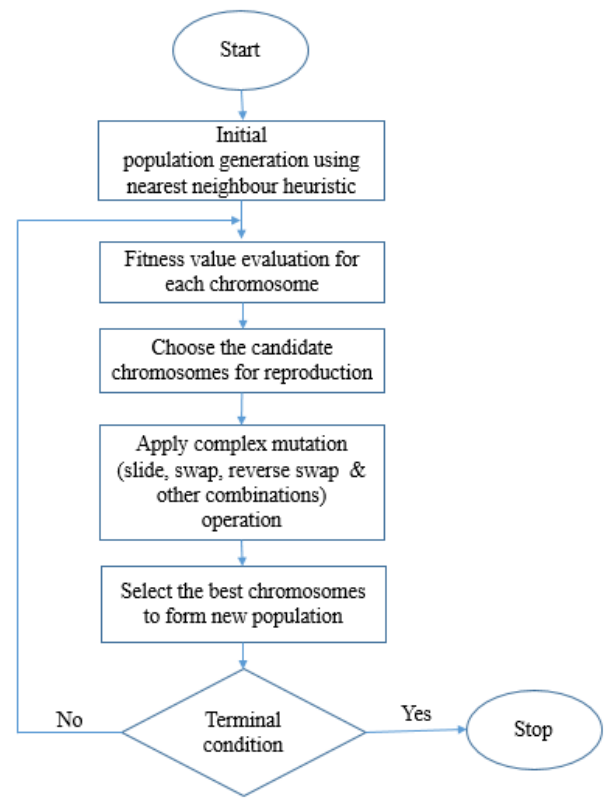

Fig. 8. Flowchart of the proposed GA

\section{Computational results}

Computational results are presented in this section. For all the experiments, the proposed GA uses roulette wheel selection, complex mutation (Swap, Reverse Swap and Slide) operators, to produce new offspring in every generation. The proposed GA is coded in MATLAB R2017a on a PC with Intel Core CPU i3 $2.00 \mathrm{GHz}$ and 4GB of Ram with Windows 10 Pro 64 bit like an Operating System. Since our nearest neighbor technique based genetic algorithm is the first solution method for OTSSP, no benchmark test instances are presented in the literature. Thus, the benchmark instances available in TSPLIB are utilized to create OTSSP instances. Overall, 50 instances have been considered from TSPLIB. These test instances were Euclidean, two-dimensional symmetric with distinct node scales, which are ranging from 14 to 318 cities. In all these instances, the first city is considered to be the home city. With each of these 50 instances, three distinct scenarios with each having a definite value for $k$ (i.e. $k=\left\lfloor\frac{n}{4}\right\rfloor, k=\left\lfloor\frac{n}{2}\right\rfloor$, and $k=\left\lfloor\frac{3 * n}{4}\right\rfloor$ ) are considered. This results in 150 test instances for the OTSSP. A comparative study is carried out on these instances. To do this, the proposed GA has been modified that fits for solving TSSP and tested all these 150 instances, best-found TSSP solutions are reported. However, the OTSSP is not the same as the TSP, typical test problems and optimal results of these may be useful to assess the proposed GA performance. To measure the performance of the proposed GA, each test instance is tested ten times independently and reports the best and worst solutions over ten independent runs. All the results were reported within the time limit of fewer than 10 minutes. Tables 1,2 and 3 report the computational results of proposed GA executed on 150 test instances. In all these tables, the first column labelled Instance denotes the name of the test instance followed by the number of cities at the end. The second and third columns labelled $n$ and $k$, respectively denote the size of the test instance (i.e. the number of cities involved in the test instance) and the number of cities required to be covered by the salesman (i.e. $k$ out of $n$ ). The fourth and fifth columns respectively denote the best known TSSP solution presented in the literature and the best found TSSP solution through proposed GA. The sixth column labelled Gap denotes the gap/deviation between the best-known TSSP and best TSSP solution produced by proposed GA. It is evaluated by using the formula (10). Here the Gap takes both positive as well as negative values. Note that positive gap values specify that the best-found TSSP solution by proposed GA is better than the best-known TSSP solution and the value zero represent that both the solutions coincide. Similarly, negative values indicate that the bestfound TSSP solution is worse than the best-known TSSP solution. Finally, columns seventh and eight labelled Best and Worst denotes the best and worst OTSSP solution produced by proposed GA, respectively.

$$
\text { Gap }=\left|\frac{\text { Best known TSSP solution-Best found TSSP solution by GA }}{\text { Best known TSSP Solution }}\right|
$$

\section{Table 1}

Computational results of proposed GA on instances with $k=\left\lfloor\frac{n}{4}\right\rfloor$ 
Instance

$n \quad k$

Best known

Best found TSSP solution TSSP solution

\begin{tabular}{|c|c|c|c|c|c|c|c|}
\hline & & & & & & Best & Worst \\
\hline a280 & 280 & 70 & 670 & 686 & -0.0239 & 606 & 606 \\
\hline bayg29 & 29 & 7 & 332 & 332 & 0 & 246 & 246 \\
\hline bays 29 & 29 & 7 & 400 & 400 & 0 & 282 & 282 \\
\hline berlin52 & 52 & 13 & 679 & 679 & 0 & 489 & 489 \\
\hline bier127 & 127 & 31 & 10619 & 10619 & 0 & 9840 & 10132 \\
\hline burma14 & 14 & 3 & 359 & 280 & 0.2201 & 151 & 151 \\
\hline $\operatorname{ch} 130$ & 130 & 32 & 1130 & 1130 & 0 & 1116 & 1119 \\
\hline $\operatorname{ch} 150$ & 150 & 37 & 1276 & 1294 & -0.0141 & 1204 & 1204 \\
\hline d198 & 198 & 49 & 5027 & 5002 & 0.005 & 3269 & 3269 \\
\hline dantzig42 & 42 & 10 & 145 & 145 & 0 & 99 & 99 \\
\hline eil101 & 101 & 25 & 107 & 107 & 0 & 101 & 101 \\
\hline eil51 & 51 & 12 & 82 & 82 & 0 & 71 & 71 \\
\hline eil76 & 76 & 19 & 102 & 102 & 0 & 99 & 99 \\
\hline fri26 & 26 & 6 & 243 & 243 & 0 & 145 & 145 \\
\hline gil262 & 262 & 65 & 540 & 540 & 0 & 509 & 509 \\
\hline gr137 & 137 & 34 & 17399 & 17399 & 0 & 14784 & 14784 \\
\hline gr17 & 17 & 4 & 234 & 234 & 0 & 143 & 143 \\
\hline gr21 & 21 & 5 & 324 & 324 & 0 & 178 & 178 \\
\hline gr24 & 24 & 6 & 264 & 264 & 0 & 231 & 231 \\
\hline gr48 & 48 & 12 & 874 & 874 & 0 & 558 & 558 \\
\hline gr96 & 96 & 24 & 10460 & 9543 & 0.0877 & 7704 & 7704 \\
\hline gr202 & 202 & 50 & 8142 & 8142 & 0 & 6977 & 6977 \\
\hline gr229 & 229 & 57 & 18555 & 18555 & 0 & 18471 & 18471 \\
\hline hk48 & 48 & 12 & 2827 & 2827 & 0 & 2094 & 2094 \\
\hline kroA100 & 100 & 25 & 4970 & 5203 & -0.0469 & 4369 & 4369 \\
\hline kroA150 & 150 & 37 & 5690 & 5690 & 0 & 5286 & 5286 \\
\hline kroA200 & 200 & 50 & 6202 & 6202 & 0 & 6138 & 6138 \\
\hline kroB100 & 100 & 25 & 4305 & 4303 & 0.0005 & 4014 & 4014 \\
\hline kroB150 & 150 & 37 & 5812 & 5812 & 0 & 5119 & 5119 \\
\hline kroB200 & 200 & 50 & 6368 & 6100 & 0.0421 & 5890 & 5890 \\
\hline kroC100 & 100 & 25 & 4964 & 4967 & -0.0006 & 4293 & 4293 \\
\hline kroD100 & 100 & 25 & 4762 & 4762 & 0 & 3991 & 3991 \\
\hline kroE100 & 100 & 25 & 3905 & 3905 & 0 & 3663 & 3663 \\
\hline $\operatorname{lin} 105$ & 105 & 26 & 2606 & 2606 & 0 & 2108 & 2108 \\
\hline $\operatorname{lin} 318$ & 318 & 79 & 8901 & 8901 & 0 & 8705 & 8705 \\
\hline pr107 & 107 & 26 & 8443 & 8443 & 0 & 6981 & 6981 \\
\hline pr124 & 124 & 31 & 14640 & 14325 & 0.0215 & 9596 & 9596 \\
\hline pr136 & 136 & 34 & 21116 & 21116 & 0 & 20928 & 20928 \\
\hline pr144 & 144 & 36 & 14327 & 14327 & 0 & 10743 & 10743 \\
\hline pr152 & 152 & 38 & 23195 & 20029 & 0.1365 & 15403 & 15403 \\
\hline pr76 & 76 & 19 & 23450 & 23450 & 0 & 17728 & 17728 \\
\hline pr226 & 226 & 56 & 20033 & 20033 & 0 & 18594 & 18645 \\
\hline rat195 & 195 & 48 & 557 & 565 & -0.0144 & 538 & 538 \\
\hline rat99 & 99 & 24 & 284 & 291 & -0.0246 & 260 & 260 \\
\hline rd100 & 100 & 25 & 1438 & 1438 & 0 & 1261 & 1261 \\
\hline St70 & 70 & 17 & 120 & 120 & 0 & 110 & 114 \\
\hline Swiss42 & 42 & 10 & 192 & 100 & 0.4792 & 66 & 66 \\
\hline U159 & 159 & 39 & 8983 & 9085 & -0.0114 & 8494 & 8494 \\
\hline Ulysses 16 & 16 & 4 & 935 & 935 & 0 & 618 & 618 \\
\hline Ulysses 22 & 22 & 5 & 747 & 747 & 0 & 447 & 447 \\
\hline
\end{tabular}


Table 2

Computational results of proposed GA on instances with $k=\left\lfloor\frac{n}{2}\right\rfloor$

\begin{tabular}{|c|c|c|c|c|c|c|c|}
\hline \multirow[t]{2}{*}{ Instance } & \multirow[t]{2}{*}{$n$} & \multirow[t]{2}{*}{$k$} & \multirow[t]{2}{*}{$\begin{array}{l}\text { Best known } \\
\text { TSSP solution }\end{array}$} & \multirow[t]{2}{*}{$\begin{array}{l}\text { Best found } \\
\text { TSSP solution }\end{array}$} & \multirow[t]{2}{*}{ Gap } & \multicolumn{2}{|c|}{$\begin{array}{l}\text { OTSSP solution by pro- } \\
\text { posed GA }\end{array}$} \\
\hline & & & & & & Best & Worst \\
\hline at280 & 280 & 140 & 1314 & 1358 & -0.0335 & 1234 & 1354 \\
\hline bayg29 & 29 & 14 & 626 & 626 & 0 & 581 & 581 \\
\hline bays 29 & 29 & 14 & 733 & 733 & 0 & 672 & 672 \\
\hline berlin52 & 52 & 26 & 1874 & 1874 & 0 & 1766 & 1766 \\
\hline Bier127 & 127 & 63 & 26062 & 26107 & -0.0017 & 24862 & 24862 \\
\hline burma14 & 14 & 7 & 1272 & 1236 & 0.0283 & 842 & 842 \\
\hline ch130 & 130 & 65 & 2408 & 2408 & 0 & 2492 & 2492 \\
\hline $\operatorname{ch} 150$ & 150 & 75 & 2761 & 2761 & 0 & 2772 & 2790 \\
\hline d198 & 198 & 99 & 7058 & 7086 & -0.0040 & 5220 & 5261 \\
\hline dantzig42 & 42 & 21 & 260 & 260 & 0 & 227 & 227 \\
\hline eil101 & 101 & 50 & 227 & 227 & 0 & 249 & 249 \\
\hline eil51 & 51 & 25 & 175 & 175 & 0 & 179 & 181 \\
\hline eil76 & 76 & 38 & 216 & 219 & -0.0139 & 217 & 219 \\
\hline fri26 & 26 & 13 & 414 & 414 & 0 & 308 & 339 \\
\hline gil262 & 262 & 131 & 1042 & 1042 & 0 & 1049 & 1049 \\
\hline gr137 & 137 & 68 & 29363 & 31784 & -0.0825 & 29108 & 29108 \\
\hline gr17 & 17 & 8 & 517 & 517 & 0 & 359 & 368 \\
\hline gr21 & 21 & 10 & 918 & 918 & 0 & 683 & 683 \\
\hline gr24 & 24 & 12 & 504 & 504 & 0 & 396 & 396 \\
\hline gr48 & 48 & 24 & 1819 & 1819 & 0 & 1691 & 1691 \\
\hline gr96 & 96 & 48 & 20688 & 19876 & 0.0392 & 17634 & 17634 \\
\hline gr202 & 202 & 101 & 14181 & 14181 & 0 & 13996 & 14131 \\
\hline gr229 & 229 & 114 & 41005 & 41005 & 0 & 41661 & 41877 \\
\hline hk48 & 48 & 24 & 4701 & 4701 & 0 & 4238 & 4300 \\
\hline kroA100 & 100 & 50 & 9184 & 10050 & -0.0943 & 9073 & 9098 \\
\hline kroA150 & 150 & 75 & 11625 & 11625 & 0 & 11412 & 11483 \\
\hline kroA200 & 200 & 100 & 12753 & 12753 & 0 & 13315 & 13315 \\
\hline kroB100 & 100 & 50 & 9096 & 9096 & 0 & 9071 & 9071 \\
\hline kroB150 & 150 & 75 & 11535 & 11535 & 0 & 11501 & 11501 \\
\hline kroB200 & 200 & 100 & 13080 & 13113 & -0.0025 & 12787 & 12787 \\
\hline kroC100 & 100 & 50 & 9457 & 9457 & 0 & 9428 & 9498 \\
\hline kroD100 & 100 & 50 & 8719 & 8719 & 0 & 8808 & 8808 \\
\hline kroE100 & 100 & 50 & 9102 & 9176 & -0.0081 & 9370 & 9370 \\
\hline $\operatorname{lin} 105$ & 100 & 52 & 5848 & 5954 & -0.0181 & 5532 & 5532 \\
\hline $\operatorname{lin} 318$ & 318 & 159 & 18600 & 19114 & -0.0276 & 17655 & 17655 \\
\hline pr107 & 107 & 53 & 18028 & 18028 & 0 & 14839 & 14839 \\
\hline pr124 & 124 & 62 & 22998 & 22998 & 0 & 21420 & 21564 \\
\hline pr136 & 136 & 68 & 46890 & 46909 & -0.0004 & 47911 & 48960 \\
\hline pr144 & 144 & 72 & 28402 & 28059 & 0.0121 & 26296 & 26296 \\
\hline pr152 & 152 & 76 & 36637 & 38863 & -0.0608 & 30712 & 30718 \\
\hline $\operatorname{Pr} 76$ & 76 & 38 & 41248 & 42638 & -0.0337 & 37793 & 37793 \\
\hline pr226 & 226 & 113 & 38718 & 39597 & -0.0227 & 33349 & 33349 \\
\hline Rat195 & 195 & 97 & 1140 & 1160 & -0.0175 & 1106 & 1108 \\
\hline rat99 & 99 & 49 & 574 & 574 & 0 & 554 & 554 \\
\hline rd100 & 100 & 50 & 3168 & 3168 & 0 & 3023 & 3203 \\
\hline St70 & 70 & 35 & 260 & 260 & 0 & 250 & 264 \\
\hline Swiss42 & 42 & 21 & 458 & 186 & 0.5939 & 132 & 141 \\
\hline U159 & 159 & 79 & 18401 & 18401 & 0 & 16750 & 17198 \\
\hline Ulysses 16 & 16 & 8 & 1685 & 1685 & 0 & 1329 & 1329 \\
\hline Ulysses 22 & 22 & 11 & 1902 & 1902 & 0 & 1473 & 1473 \\
\hline
\end{tabular}


Table 3

Computational results of proposed GA on instances with $k=\left\lfloor\frac{3 * n}{4}\right\rfloor$

\begin{tabular}{|c|c|c|c|c|c|c|c|}
\hline \multirow[t]{2}{*}{ Instance } & \multirow[t]{2}{*}{$n$} & \multirow[t]{2}{*}{$k$} & \multirow[t]{2}{*}{ Best known TSSP solution } & \multirow[t]{2}{*}{ Best found TSSP solution } & \multirow[t]{2}{*}{ Gap } & \multicolumn{2}{|c|}{ OTSSP solution by proposed GA } \\
\hline & & & & & & Best & Worst \\
\hline at280 & 280 & 210 & 2066 & 2094 & -0.0136 & 1894 & 1925 \\
\hline bayg29 & 29 & 21 & 999 & 999 & 0 & 929 & 929 \\
\hline bays 29 & 29 & 21 & 1194 & 1194 & 0 & 1090 & 1090 \\
\hline berlin52 & 52 & 39 & 4174 & 4174 & 0 & 3853 & 3904 \\
\hline Bier127 & 127 & 95 & 50324 & 50764 & -0.0087 & 51542 & 51604 \\
\hline burma14 & 14 & 10 & 1642 & 1575 & 0.0408 & 1349 & 1349 \\
\hline $\operatorname{ch} 130$ & 130 & 97 & 3907 & 4158 & -0.0642 & 4062 & 4127 \\
\hline $\operatorname{ch} 150$ & 150 & 112 & 4499 & 4720 & -0.0491 & 4480 & 4480 \\
\hline d198 & 198 & 148 & 9386 & 9363 & 0.0025 & 7874 & 7874 \\
\hline dantzig42 & 42 & 31 & 427 & 427 & 0 & 404 & 404 \\
\hline eillo1 & 101 & 75 & 396 & 406 & -0.0253 & 398 & 398 \\
\hline eil51 & 51 & 38 & 289 & 287 & 0.0069 & 278 & 286 \\
\hline eil76 & 76 & 57 & 336 & 336 & 0 & 345 & 348 \\
\hline fri26 & 26 & 19 & 601 & 601 & 0 & 492 & 492 \\
\hline gil262 & 262 & 196 & 1672 & 1695 & -0.0138 & 1671 & 1671 \\
\hline gr137 & 137 & 102 & 43912 & 48623 & -0.1073 & 44147 & 44286 \\
\hline gr17 & 17 & 12 & 951 & 951 & 0 & 640 & 640 \\
\hline gr21 & 21 & 15 & 1501 & 1501 & 0 & 1276 & 1276 \\
\hline gr24 & 24 & 18 & 844 & 844 & 0 & 763 & 763 \\
\hline gr48 & 48 & 36 & 3104 & 3104 & 0 & 3135 & 3135 \\
\hline gr96 & 96 & 72 & 31437 & 31095 & 0.0109 & 29257 & 30083 \\
\hline gr202 & 202 & 151 & 21563 & 21954 & -0.0181 & 22000 & 22528 \\
\hline gr229 & 229 & 171 & 69201 & 67848 & 0.0196 & 65832 & 66183 \\
\hline hk48 & 48 & 36 & 7278 & 7278 & 0 & 6937 & 6937 \\
\hline kroA100 & 100 & 75 & 14492 & 14492 & 0 & 13982 & 14229 \\
\hline kroA150 & 150 & 112 & 18210 & 18295 & -0.0047 & 17787 & 17953 \\
\hline kroA200 & 200 & 150 & 20723 & 20135 & 0.0284 & 20705 & 20855 \\
\hline kroB100 & 100 & 75 & 14744 & 14744 & 0 & 14648 & 14787 \\
\hline kroB150 & 150 & 112 & 17501 & 17349 & 0.0087 & 17090 & 17193 \\
\hline kroB200 & 200 & 150 & 20508 & 21266 & -0.0370 & 20553 & 20851 \\
\hline kroC100 & 100 & 75 & 14067 & 14067 & 0 & 14295 & 14419 \\
\hline kroD100 & 100 & 75 & 14171 & 14171 & 0 & 13884 & 14120 \\
\hline kroE100 & 100 & 75 & 14640 & 14640 & 0 & 15347 & 15347 \\
\hline $\operatorname{lin} 105$ & 105 & 78 & 9034 & 8999 & 0.0039 & 8412 & 8412 \\
\hline $\operatorname{lin} 318$ & 318 & 238 & 29829 & 29829 & 0 & 27963 & 28051 \\
\hline pr107 & 107 & 80 & 36468 & 37605 & -0.0312 & 29684 & 29684 \\
\hline pr124 & 124 & 93 & 39174 & 39174 & 0 & 36977 & 36977 \\
\hline pr136 & 136 & 102 & 69690 & 70790 & -0.0158 & 67879 & 69818 \\
\hline pr144 & 144 & 108 & 41452 & 41703 & -0.0061 & 40274 & 40832 \\
\hline pr152 & 152 & 114 & 57431 & 52393 & 0.0877 & 46434 & 46635 \\
\hline Pr76 & 76 & 57 & 64142 & 64918 & -0.0121 & 62262 & 62262 \\
\hline pr226 & 226 & 169 & 47516 & 49198 & -0.0354 & 49022 & 49588 \\
\hline rat195 & 195 & 146 & 1753 & 1713 & 0.0228 & 1669 & 1670 \\
\hline rat99 & 99 & 74 & 861 & 870 & -0.0105 & 868 & 868 \\
\hline rd100 & 100 & 75 & 5094 & 5175 & -0.0159 & 5476 & 5476 \\
\hline St70 & 70 & 52 & 428 & 428 & 0 & 437 & 446 \\
\hline Swiss42 & 42 & 31 & 760 & 333 & 0.5618 & 281 & 281 \\
\hline U159 & 159 & 119 & 27413 & 27612 & -0.0073 & 27342 & 27348 \\
\hline Ulysses 16 & 16 & 12 & 3183 & 3183 & 0 & 2704 & 2704 \\
\hline Ulysses 22 & 22 & 16 & 2941 & 2968 & -0.0092 & 2618 & 2618 \\
\hline
\end{tabular}

In Table 1, overall, 50 test instances were tested using the proposed GA with $k=\left\lfloor\frac{n}{4}\right\rfloor$. To measure the performance of the proposed GA, the best-found TSSP solutions are compared with the best-known TSSP solutions available in the literature. It 
is seen that the number of test instances for which the proposed GA produced solutions coincide with the best-known solutions is 35 out of 50. The number of test instances for which the proposed GA provided solutions worse than the best known are 7 out of 50. Whereas, the number of test instances for which the best-known results improved is 8 out of 50. It is also seen that the negative Gap values vary from -0.0469 to -0.006 . Whereas positive Gap values are ranging from 0.0005 to 0.4792 . Furthermore, the best and worst found OTSSP solutions for all the 50 instances generated by the proposed GA are also reported. Similarly, in Table 2, the same test instances were used as used in Table 1 but with $k=\left\lfloor\frac{n}{2}\right\rfloor$. From Table 2, it is evident that the number of test instances for which the proposed GA provided solutions coincides with the best-known solutions is 31 out of 55. The number of test instances for which the produced solutions worse than the best known are 15 out of 55 . Whereas, the number of test instances for which the best-known results improved is 4 out of 55. It is also seen that the negative Gap values vary from -0.0943 to -0.0004 , whereas positive Gap values range from 0.0121 to 0.5939 . Besides, the best and worst found OTSSP solutions for all these 50 test instances are presented. Finally, in Table 3, the same test instances were used but with $k=\left\lfloor\frac{3 * n}{4}\right\rfloor$.

From Table 3, It is observed that the number of test instances for which the obtained results coincides and worse than the bestknown results are 20 and 19 out of 50, respectively. The number of test instances for which the best-known results improved is 11 out of 50 . It is also seen that the negative Gap values vary from $-0.1073 \%$ to -0.0047 , whereas positive Gap values range from 0.0025 to 0.5618 . Further, the best and worst found OTSSP solutions for these entire 50 test instances tested on proposed GA are also presented. From overall computational results, it is seen that the proposed GA is having a great capability in providing the best TSSP solutions. With this capability, the proposed GA will certainly provide the best solutions for OTSSP in a limited time. Note that 23 out of 150 test cases has improved solutions, which are reported in boldface. These new solutions will be useful as a reference to future comparative studies. Although the structure of TSSP and OTSSP are almost similar with simple relaxation, the optimal solution for TSSP may become the worse solution for OTSSP after removal of edge from the last city to home city. Similarly, the optimal solution for OTSSP may results worse solution for TSSP after including an edge from the last city of the route plan to home city. To show the variation in route plans and its traversal distances as per the structure of the model (TSSP and OTSSP) and different $k$ values, a simple test instance namely burmal4 with 14 cities has been considered. Fig. 9 demonstrates these plots. In Fig. 9, plots ( $a, b$ and c) represent the TSSP solutions, whereas plots ( $\mathrm{d}$, e, and $\mathrm{f}$ ) denote the OTSSP solutions generated by the proposed GA with distinct $k$ values $(3,7,10)$. In all the plots of this figure, the cities are represented with star symbols labelled with respective city numbers, the home city where the salesman starts and ends his tour is shown with a diamond symbol. This figure clearly shows that the difference in route plans and its traversal distance for the TSSP and OTSSP as per the $k$ value.

\section{Conclusions}

In this study, we have developed a hybrid algorithm that comprises the nearest neighbor technique and crossover free genetic algorithm with complex mutation operators for the OTSSP. The developed algorithm effectively deals with both the features of OTSSP namely, subset selection and permutation. To best of the author's knowledge, this is the first hybrid GA for the OTSSP. As there are no existing benchmark instances for OTSSP, several OTSSP test instances are generated from the TSPLIB to assess the effectiveness of the algorithm proposed. The computational results demonstrate that the proposed algorithm is having great potential in achieving the best results for the OTSSP. However, the physical structure of TSSP and OTSSP models looks similar with simple relaxation (removal of an edge from the last city to home city), but these models are independent to one another. This means that the optimal solution of TSSP may not provide the optimal solution for OTSSP by just deleting an edge from the last city of the route plan to the home city. As the proposed approach is the first GA for OTSSP, this algorithm will be served as the reference approach for measuring the performance of forthcoming heuristic, metaheuristic and hybrid algorithms. Solution techniques analogous to our algorithm can be designed for other models by incorporating effective strategies as per the features of the model. In future, we aim to develop hybrid genetic algorithms for distinct variants of the TSP. 


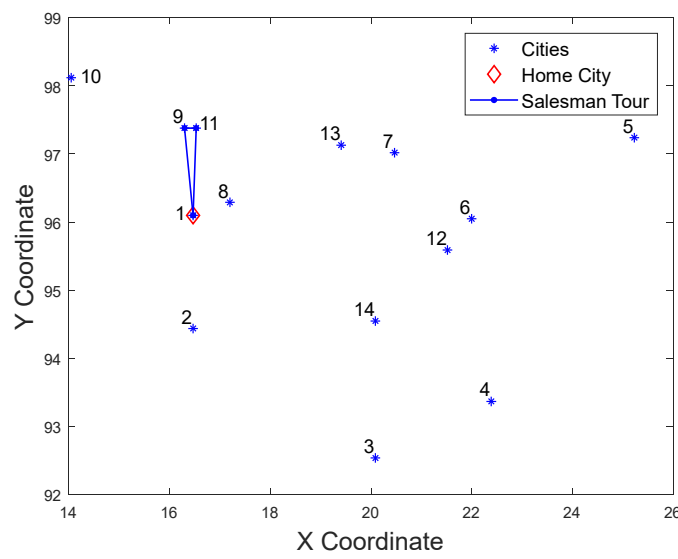

(a) $k=3 \&$ distance $=280$

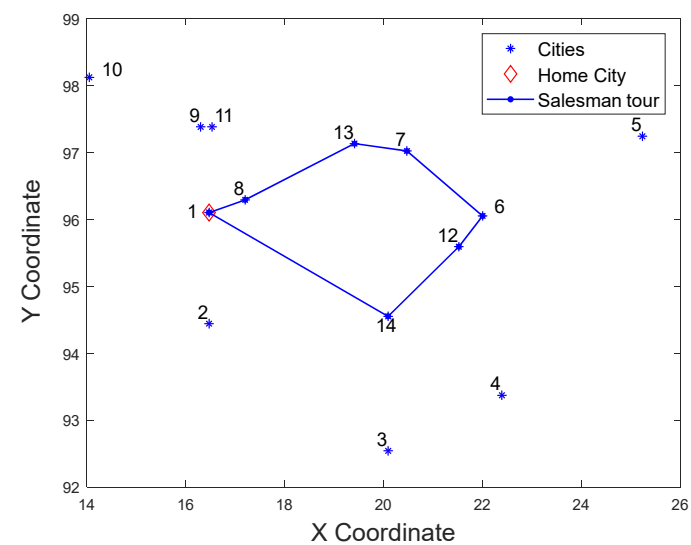

(b) $k=7 \&$ distance $=1236$

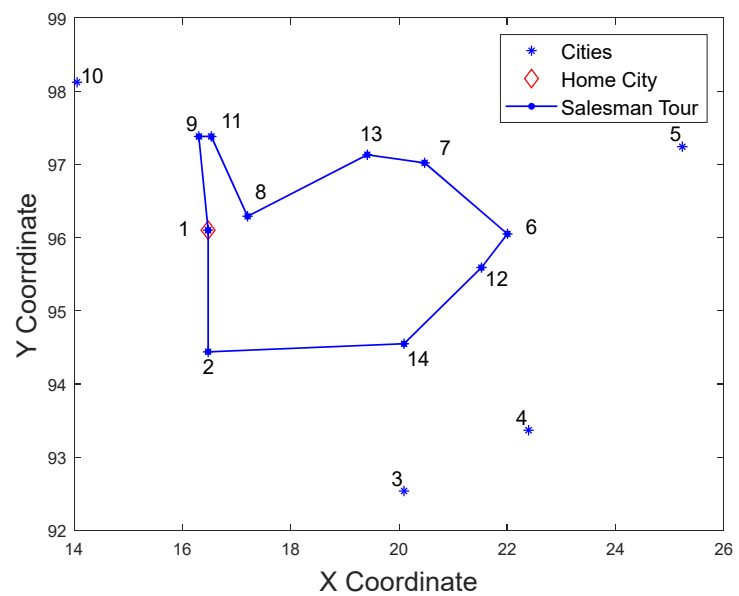

(c) $k=10 \&$ distance $=1575$

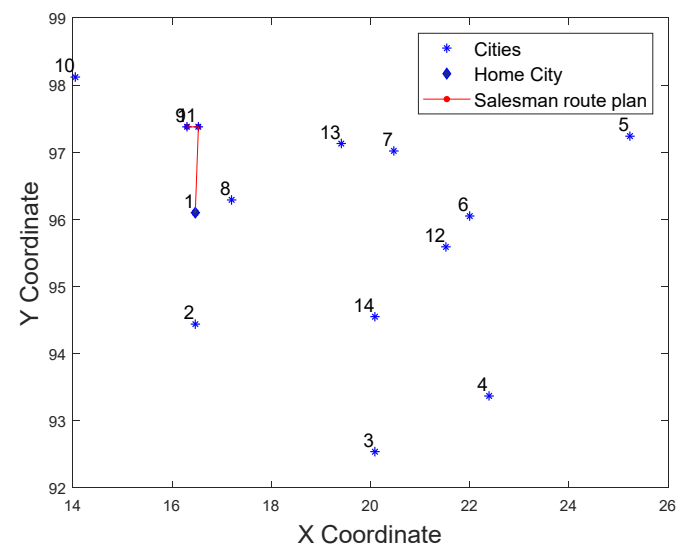

(d) $k=3 \&$ distance $=151$

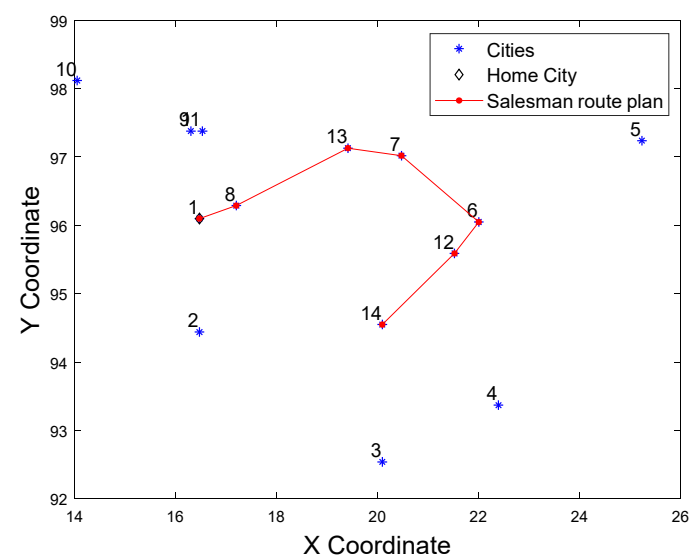

(e) $k=7 \&$ distance $=842$

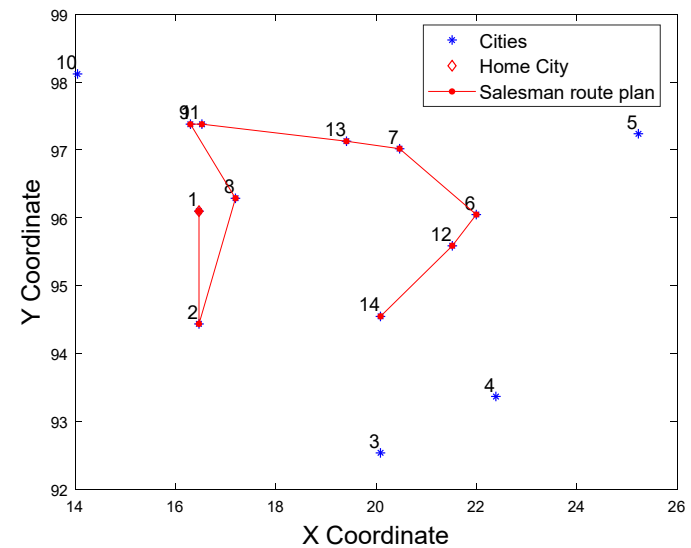

(f) $k=10 \&$ distance $=1349$

Fig.9. Best found TSSP $(\mathrm{a}, \mathrm{b}, \mathrm{c})$ and $\operatorname{OTSSP}(\mathrm{d}, \mathrm{e}, \mathrm{f})$ solutions by proposed GA on burma14 with distinct $k$ values. 


\section{References}

Ausiello, G., Bonifaci, V., Leonardi, S., Marchetti-Spaccamela, A., \& Gonzalez, T. F. (2018). Prize Collecting Traveling Salesman and Related Problems.

Bahaabadi, M.R., Mohaymany, A.S., \& Babaei, M. (2012). An Efficient crossover operator for travelling salesman problem. International Journal of Optimization in Civil Engineering 2(4), 607-619.

Balas, E. (1989). The prize collecting traveling salesman problem. Networks, 19(6), 621-636.

Chieng, H. H., \& Wahid, N. (2014). A performance comparison of genetic algorithm's mutation operators in n-cities open loop travelling salesman problem. In Recent Advances on Soft Computing and Data Mining (pp. 89-97). Springer, Cham.

Gensch, D. H. (1978). An industrial application of the traveling salesman's subtour problem. AIIE Transactions, 10(4), 362370.

Giardini, G., \& Kalmar-Nagy, T. (2011). Genetic algorithm for combinatorial path planning: the subtour problem. Mathematical Problems in Engineering, 2011.

Goldenberg, D. E. (1989). Genetic algorithms in search, optimization and machine learning. Addison-Wesley, Newyork.

Hussain, A., Muhammad, Y. S., Nauman Sajid, M., Hussain, I., Mohamd Shoukry, A., \& Gani, S. (2017). Genetic algorithm for traveling salesman problem with modified cycle crossover operator. Computational intelligence and neuroscience, 2017.

Ibaraki, T. (1973). Algorithms for obtaining shortest paths visiting specified nodes. Siam Review, 15(2), 309-317.

Laporte, G., Mercure, H., \& Norbert, Y. (1984). Optimal tour planning with specified nodes. RAIRO-Operations ResearchRecherche Opérationnelle, 18(3), 203-210.

Liu, C., \& Kroll, A. (2016). Performance impact of mutation operators of a subpopulation-based genetic algorithm for multirobot task allocation problems. SpringerPlus, 5(1), 1361.

Maredia, A., \& Pepper, R. (2010). History, Analysis, and Implementation of Traveling Salesman Problem (TSP) and Related Problems. Department of Computer and Mathematical Sciences, University of Houston-Downtown.

Matai, R., Singh, S. P., \& Mittal, M. L. (2010). Traveling salesman problem: an overview of applications, formulations, and solution approaches. Traveling salesman problem, theory and applications, 1 .

Mittenthal, J., \& Noon, C. E. (1992). An insert/delete heuristic for the travelling salesman subset-tour problem with one additional constraint. Journal of the Operational Research Society, 43(3), 277-283.

Pandiri, V., \& Singh, A. (2020). Two multi-start heuristics for the k-traveling salesman problem. OPSEARCH, 57(4), 11641204.

Saksena, J. P., \& Kumar, S. (1966). The routing problem with "K” specified nodes. Operations Research, 14(5), 909-913.

Stetsyuk, P. I. (2016). Problem statements for k-node shortest path and k-node shortest cycle in a complete graph. Cybernetics and Systems Analysis, 52(1), 71-75.

Venkatesh, P., Srivastava, G., \& Singh, A. (2018). A general variable neighborhood search algorithm for the k-traveling salesman problem. Procedia computer science, 143, 189-196.

Verweij, B., \& Aardal, K. (2003). The merchant subtour problem. Mathematical programming, 94(2-3), $295-322$.

Westerlund, A., Göthe-Lundgren, M., \& Larsson, T. (2006). A stabilized column generation scheme for the traveling salesman subtour problem. Discrete Applied Mathematics, 154(15), 2212-2238. 
(C) 2020 by the authors; licensee Growing Science, Canada. This is an open access article distributed under the terms and conditions of the Creative Commons Attribution (CC-BY) license (http://creativecommons.org/licenses/by/4.0/). 